\title{
Lung neoplasm diagnostics using Raman spectroscopy and autofluorescence analysis
}

\author{
V.P. Zakharov ${ }^{1 *}$, I.A. Bratchenko ${ }^{1}$, D.N. Artemyev $^{1}$, O.O. Myakinin ${ }^{1}$, D.V. Kornilin ${ }^{1}$, S.V. Kozlov ${ }^{2}$, \\ A.A. Moryatov ${ }^{2}$ \\ ${ }^{1}$ Laser and biotechnical systems department, Samara State Aerospace University, 34 Moskovskoe Shosse, Samara, \\ 443086, Russia \\ ${ }^{2}$ Oncology Department, Samara State Medical University, 89 Chapaevskaya str., Samara, 443099, Russia \\ *e-mail: zakharov@ssau.ru
}

\begin{abstract}
A method of lung neoplasm diagnostics is proposed based on the combined analysis of Raman and autofluorescence spectra excited by laser beam with the wavelength $785 \mathrm{~nm}$. Such approach allows fast selection of the pathology region followed by differentiation of the neoplasm type. The developed method demonstrated the sensitivity of $90.9 \%$ and the specificity of $71.4 \%$ in the differentiation of squamous cell carcinoma and adenocarcinoma cases. (C) 2015 Samara State Aerospace University (SSAU).
\end{abstract}

Keywords: Raman spectroscopy; autofluorescence; optical diagnostics for medicine; spectroscopy; biological tissue; lung cancer.

Paper \#1988 received 2014.12.11; revised manuscript received 2015.01.20; accepted for publication 2015.01.20; published online 2015.03.28.

\section{References}

1. P. Boyle, World Cancer Report 2008, International Agency for Research on Cancer, Lyon (2008).

2. C. D. Mathers, and D. Loncar, "Projections of global mortality and burden of disease from 2002 to 2030 ," PLoS Med 3. e442 (2006).

3. R. J. Friedman, "The diagnostic performance of expert dermatoscopics vs a computer-vision system on small diameters melanomas," Arch Dermatol. 144(4), 476-482 (2008).

4. J. Zhao, H. Lui, D.I. McLean, and H. Zeng, "Real-time Raman spectroscopy for noninvasive in vivo skin analysis and diagnosis," New developments in biomedical engineering 24, 455-474 (2010).

5. Z. Huang, A. McWilliams, H. Lui, D. I. McLean, S. Lam, and H. Zeng, "Near-infrared Raman spectroscopy for optical diagnosis of lung cancer," Int J Cancer. 107(6), 1047-1052 (2003).

6. J. Surmacki, J. Musial, R. Kordek, and H. Abramczyk, "Raman imaging at biological interfaces: Applications in breast cancer diagnosis," Molecular Cancer, 12(1), 48 (2013).

7. P. C. Ashok, B. B. Praveen, N. Bellini, A. Riches, K. Dholakia, and C. S. Herrington, "Multi-modal approach using Raman spectroscopy and optical coherence tomography for the discrimination of colonic adenocarcinoma from normal colon," Biomedical Optics Express 4(10), 2179-2186 (2013).

8. A. Pavićević, S. Glumac, J. Sopta, A. Popović-Bijelić, M. Mojović, and Goran Bačić, "Raman microspectroscopy as a biomarking tool for in vitro diagnosis of cancer: a feasibility study," Croat Med J. 53, 551-557 (2012).

9. J. Zhao, H. Lui, D. I. McLean, and H. Zeng, "Real-time Raman spectroscopy for in vivo skin cancer diagnosis," Cancer Res. 72(10), 2491-2500 (2012).

10. N. Bulgakova, V. Sokolov, L. Telegina, K. Vereshchagin, G. Frank, and V. Chissov, "Study of laser-induced autofluorescence emission spectra from normal and malignant bronchial epithelium," Photonics and Lasers in Medicine 2(2), 93-99 (2013).

11. H. Pahlevaninezhad, A. M. D. Lee, S. Lam, C. MacAulay, and P. M. Lane, "Coregistered autofluorescenceoptical coherence tomography imaging of human lung sections," Journal of Biomedical Optics, 19(3), 036022 (2014). 
12. M. E. Darvin, N. N. Brandt, and J. Lademann, "Photobleaching as a method of increasing the accuracy in measuring carotenoid concentration in human skin by Raman spectroscopy," Optics and Spectroscopy (English translation of Optika i Spektroskopiya), 109(2), 205-210 (2010).

13. R. O. Duda, P. E. Hart, and D. G. Stork, Pattern Classification, 2nd Edition, Wiley (2000).

14. O. Yusuke, S. Hideyuki, T. Tatsuji, F. Chie, and S. Hidetoshi, "A Discrimination analysis of human lung cancer cells associated with histological type and malignancy using Raman spectroscopy," Journal of Biomedical Optics 15(1), 017009 (2010).

15. J. L. Castro, M. R. Lopez Ramirez, J. F. Arenas, and J. C. Otero, "Vibrational spectra of 3-phenylpropionic acid and L-phenylalanine," Journal of Molecular Structure 887, 744-747 (2005).

16. C. De Silva, K. Czarnecki, and M.D. Ryan, "Visible and resonance Raman spectra of low valent iron porphyrins," Inorganica Chimica Acta 287, 21-26 (1999).

17. C.-H. Chuang, and Y.-T. Chen, "Raman scattering of L-tryptophan enhanced by surface plasmon of silver nanoparticles: vibrational assignment and structural determination," J. Raman Spectrosc. 40, 150-156 (2009).

18. C. Magnon, S. J. Hall, J. Lin, X. Xue, L. Gerber, S. J. Freedland, and P.S. Frenette, "Autonomic Nerve Development Contributes to Prostate Cancer Progression," Science 341(6142), 1236361 (2013).

19. A. F. D'Adamo Jr., and F. M. Yatsu, "Acetate metabolism in the nervous system," Journal of Neurochemistry 13(10), 961-965 (1966).

20. A. Barth, "The infrared absorption of amino acid side chains," Progress in Biophysics \& Molecular Biology. 74, 141-173 (2000).

21. P. Kupser, K. Pagel, J. Oomens, N. Polfer, B. Koksch, G. Meijer, and G. von Helden, "Amide-I and -II Vibrations of the Cyclic $\beta$-Sheet Model," J. Am. Chem. Soc. 132(6), 2085-2093 (2010).

22. M. Keller, E. M. Kanter, and A. Mahadevan-Jansen, "Raman spectroscopy for cancer diagnosis" Spectroscopy 21(11), 33-41 (2006).

23. I. J. Bigio, and J. R. Mourant, “Optical Biopsy,” Encyclopedia of Optical Engineering, 1577-1593 (2003).

24. Y. Freund, and R.E. Schapire, "A Decision-Theoretic Generalization of On-Line Learning and an Application to Boosting," Journal of Computer and System Sciences 55(1), 119-139 (1997).

25. N. Otsu, "A threshold selection method from gray-level histograms," IEEE Trans. Sys. Man. Cyber. 9, 62-66 (1979).

\section{Introduction}

In recent $20-25$ years the number of newly-revealed malignant and benign neoplasms of skin, lung, intestine, and internals significantly increased [1]. According to the prognosis of medical experts, the number of oncological patients will steadily increase [2]. The efficiency indicators in the early diagnostics of malignant neoplasms by general practitioners, in general, remain unsatisfactory, which is due to the complexity of interpreting the clinical differential features of neoplasms at the early stage [3]. The low level of early diagnostics of cancer diseases favours the neoplasm development and, finally, increases the risks of fatal outcome. In order to improve the efficiency of diagnostics, it is necessary to use instrumental methods and means.

One of the convenient methods of noninvasive analysis of neoplasms is Raman spectroscopy (RS). The use of this method for diagnostics of cancer neoplasms was demonstrated by a number of authors in ex vivo and in vivo studies of skin [4], lung [5], breast [6], intestine [7] and other tissues. Thus, e.g., the authors of Ref. [5] demonstrated high efficiency of Raman scattering method in lung cancer diagnostics; the achieved sensitivity was $94 \%$ and the specificity $92 \%$. However, in the case of mass screening the sensitivity and the specificity of the method are essentially lower, in particular, in the lung studies [8] the accuracy of Raman analysis of neoplasms was only $77 \%$. Similar trend is seen in the studies of other biological tissues, e.g., the skin. In Ref. [9] the achieved sensitivity and specificity are at the level of $91 \%$ and $75 \%$, respectively. The low specificity is related to the high variance of Raman scattering intensity peaks depending on the concentration of the tissue biochemical components in different samples and the use of absolute criteria in the methods, proposed in the papers considered above.

To overcome these difficulties, in this paper we propose to complete the method of Raman spectroscopy with the analysis of autofluorescence that inevitably arises under the illumination of the studied tissue with the probing laser radiation. To reduce the effect of spectral characteristics variance in the studies of various biological objects it is reasonable to use the comparative analysis of the autofluorescence and Raman scattering intensity from the tumor and from the adjacent healthy tissue.

Autofluorescence (AF) spectroscopy can be used to detect lung pathologies $[10,11]$. It is known that the biological tissues contain fluorescent chromophores: elastin, collagen, and NADH, which contribute to the autofluorescence spectrum, as well as purely absorbing chromophores, such as haemoglobin. As a rule, for AF diagnostics the lasers of UV spectral range (300-400 $\mathrm{nm})$, or those of the visible spectral range (400-650 nm) are used. The choice of these ranges is determined by the position of the basic emission spectra of the tissue chromophores. In this paper we report the analysis of 
the possibility to diagnose lung neoplasms using the intrinsic fluorescence, excited by the laser radiation with the wavelength $785 \mathrm{~nm}$.

Simultaneous application of two spectral methods provides the increase of informative features and, therefore, is expected to reduce the number of diagnostic errors, while the comparative spectral analysis will help to allow for the personal specificity of the chemical composition of the tissue under study. Besides that, the analysis of autofluorescence allows the selection of spectral anomaly regions and the fast determination of the neoplasm boundaries.

\section{Materials and methods}

\subsection{Experimental setup}

In the present work we used the experimental setup schematically presented in Fig. 1. The main elements of the setup are the following: the thermally stabilised semiconductor laser 1 (LML-785.0RB-04), the optical Raman unit 2 (PBL 785), the spectrograph 3 (Sharmrock SR-303i) with the integrated digital camera (ANDOR DV-420A-OE), and the computer 4. The probing laser radiation with the wavelength $785 \mathrm{~nm}$ via the band-pass filter BPF is directed by the mirror $\mathrm{M}$ and dichroic reflector DM, and focused by the lens $\mathrm{L}_{2}$ onto the studied sample. The filter BPF serves to filtering the incident radiation from the Raman components that might be generated in the transmitting fibre. The scattered and autofluorescence radiation is collected by the lens $\mathrm{L}_{2}$ and directed into the spectrometer 3 via the long-pass filter LPF, aimed at eliminating the laser radiation from the recorded signal. The lenses $L_{1}$ and $L_{2}$ are used for matching the optical unit 2 with the supplying and the signal optical fibres.

Raman and AF spectra were registered simultaneously with one optical unit 2 . The optical unit 2 was located directly above the studied region at the distance of 6-7 mm. The diameter of the laser probing beam was $1 \mathrm{~mm}$. Series of spectra were registered from the neoplasm and the healthy tissue. The obtained data was analysed by comparing with the results of the histologic study. The time of examination of each sample did not exceed 3-5 min. Time of signal registration was chosen to match the AF decrease conditions. Irradiation of tissue samples with exposure times about 2-3 minutes leads to the decreasing in AF level. The decrease in the intensity of AF associated with the effect of tissues photobleaching [12]. At this regard it is important to say that the intensity of tissues Raman bands remains unchanged. Thus such an approach helps to increase signal-to-noise ratio and precisely register a Raman signal.

Selection of the autofluorescence and Raman scattering signals was carried out using the method of polynomial approximation with additional filtration of random noise effects and automatic determination of the polynomial power for the given spectral interval. The polynomial fitting function corresponding to the AF was subtracted from raw acquired spectrum to separate Raman spectrum. The registered signal, which consists of Raman and AF signals, was recorded the spectral interval $800-920 \mathrm{~nm} \quad\left(250-1800 \mathrm{~cm}^{-1}\right)$ with the resolution $0.05 \mathrm{~nm}\left(1 \mathrm{~cm}^{-1}\right)$ at low level of intrinsic noises. The Raman signal was analysed in the spectral interval $1200-1800 \mathrm{~cm}^{-1}$. The AF signal was analysed in the spectral interval $250-1800 \mathrm{~cm}^{-1}$.

\subsection{Tissue samples}

In the series of ex vivo experiments the spectra were recorded from 46 samples (23 adenocarcinomas, 18 squamous cell carcinomas, and 5 carcinoids). The detailed information about the studied samples, including the information about the patients and the localisation of lung lesions, is presented in Table 1. All samples were obtained after surgical resection in Samara Regional Clinical Oncology Dispensary (SRCOD). The tissue samples were kept in sterile boxes at $+4 \pm 2{ }^{\circ} \mathrm{C}$ and were tested not later than in four hours after the resection. Each tumor examination was accompanied by histologic analysis that determined the final diagnosis.

All patients (12 women and 34 men, all Europeans) that took part in the examination were more than 18 years old, passed the preliminary examination and were prepared for the surgical treatment in the departments of SRCOD. All human tissue samples were studied in correspondence with the protocol affirmed by the Bioethics Committee of Samara State Medical University. The participation in the study was voluntary and accompanied with informed consent.

\subsection{Separation of classes in the phase plane}

To separate the classes in the phase space formed by the values of the Raman spectral peak intensities the canonical discriminant analysis (DA) was used. The quality of the approach was characterised by its sensitivity and specificity. The DA is capable of flexible change of priority in favour of the sensitivity or, on the contrary, in favour of the specificity. The DA is implemented in most of the widely used mathematical software packages.

In the present paper we use the method of quadratic discriminant analysis (QDA) [13]. In fact, the QDA determines the hyperquadric, the form of which depends upon the degree of dispersion of the experimental results and the centres of gravity of the separated classes. By varying the class priorities one can shift the dividing curve to achieve the required proportion between the sensitivity and specificity. 


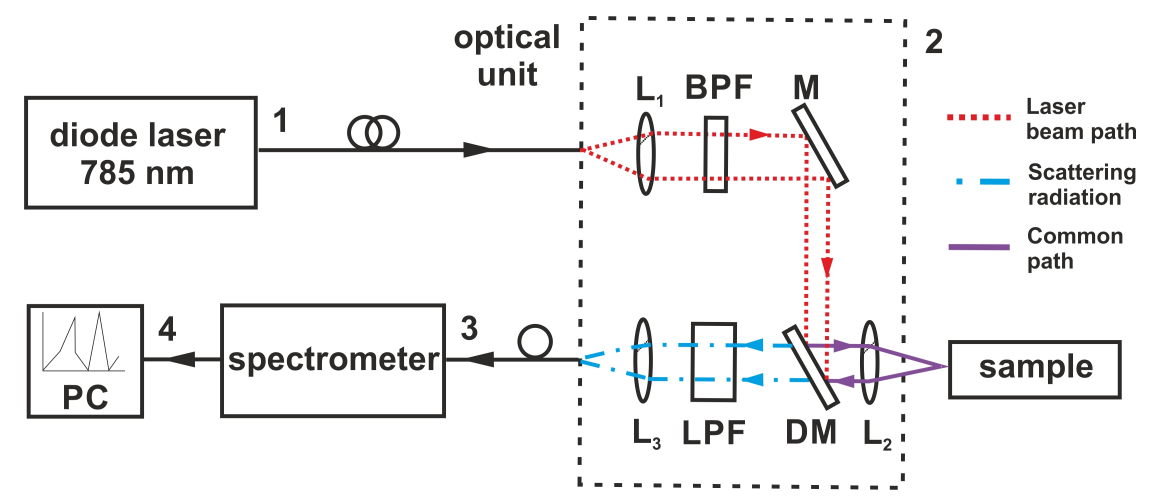

Fig. 1 Optical schematic diagram of the experimental setup.

Table 1 Summary of patients and lesions.

\begin{tabular}{llllllll}
\hline Subjects & \multicolumn{1}{c}{ Location } \\
\hline $\begin{array}{l}\text { Final lesion } \\
\text { diagnosis }\end{array}$ & $\begin{array}{l}\text { Mean age, y } \\
\text { (range) }\end{array}$ & $\begin{array}{l}\text { Mean } \\
\text { diameter, } \\
\text { cm (range) }\end{array}$ & Male & Female & $\begin{array}{l}\text { Number } \\
\text { of lesions }\end{array}$ & Right lung & Left lung \\
\hline SCC & $64(55-72)$ & $4.5(2.5-8)$ & 18 & - & 18 & 10 & 8 \\
\hline ADC & $63(42-79)$ & $2,4(0.2-6)$ & 13 & 10 & 23 & 9 & 14 \\
\hline Carcinoid & $60(57-66)$ & $2,1(0,5-4)$ & 3 & 2 & 5 & 3 & 2 \\
\hline
\end{tabular}

\section{Results and discussion}

\subsection{Analysis of Raman spectra of lung neoplasms}

The characteristic normalised Raman spectra of neoplasms and healthy lung tissue are presented in Fig. 2. The maximum of the Raman spectrum corresponds to the frequency shift by $1450 \mathrm{~cm}^{-1}$. This is the frequency of bending mode (deformation vibration) of the bonds $\mathrm{CH}_{2} / \mathrm{CH}_{3}$ that occurs in phospholipids and proteins, such as collagen. Alongside with this band, other bands are also confidently recorded, namely, the band at $1210 \mathrm{~cm}^{1}$ (stretching vibrations $\mathrm{C}-\mathrm{C}_{6} \mathrm{H}_{5}$ in tryptophan and phenylalanine), $1230 \mathrm{~cm}^{-1}$ (stretching asymmetric vibrations of $\mathrm{PO}_{2^{-}}$in nucleic acids), $1265 \mathrm{~cm}^{-1}$ (stretching vibrations of $\mathrm{CN}$, bending vibrations of $\mathrm{NH}$ in amid III, $\alpha$-spirals, collagen, and tryptophan), 1300 $\mathrm{cm}^{-1}$ (twisting and wagging $\mathrm{CH}_{2}$ vibrations in collagen and phospholipids), $1335 \mathrm{~cm}^{-1}$ (wagging vibrations of $\mathrm{CH}_{3} \mathrm{CH}_{2}$ in collagen and nucleic acids), 1520, 1550, $1580 \mathrm{~cm}^{-1}$ (stretching vibrations $\mathrm{C}=\mathrm{C}$ in carotenoids, tryptophan and porphyrin, and phenylalanine, respectively), $1620 \mathrm{~cm}^{-1}$ (stretching vibrations $\mathrm{C}=\mathrm{C}$ in tryptophan and porphyrin), $1660 \mathrm{~cm}^{-1}$ (stretching vibrations $\mathrm{C}=\mathrm{O}$ in amid $\mathrm{I}, \alpha$-spirals and proteins, such as collagen and elastin, stretching vibrations $\mathrm{C}=\mathrm{C}$ in fats), 1700,1750 and $1780 \mathrm{~cm}^{-1}$ (stretching vibrations $\mathrm{C}=\mathrm{O}$ in phospholipids) [5, 14-17].

In the ex vivo spectra of healthy lung tissue and neoplasms 6 strongest Raman bands were chosen, namely, 1200-1240, 1300-1340, 1440-1460, 1540-1580, $1650-1670$, and $1750-1790 \mathrm{~cm}^{-1}$. The normalisation of the spectra was performed using the peak value at 1450 $\mathrm{cm}^{-1}$, having the maximal intensity among the recorded peaks. The band $1750-1790 \mathrm{~cm}^{-1}$ has the smallest intensity, and the relative intensity of this peak for the healthy tissue slightly differs from the analogous value in the region of tumor both for adenocarcinoma and for squamous cell carcinoma.

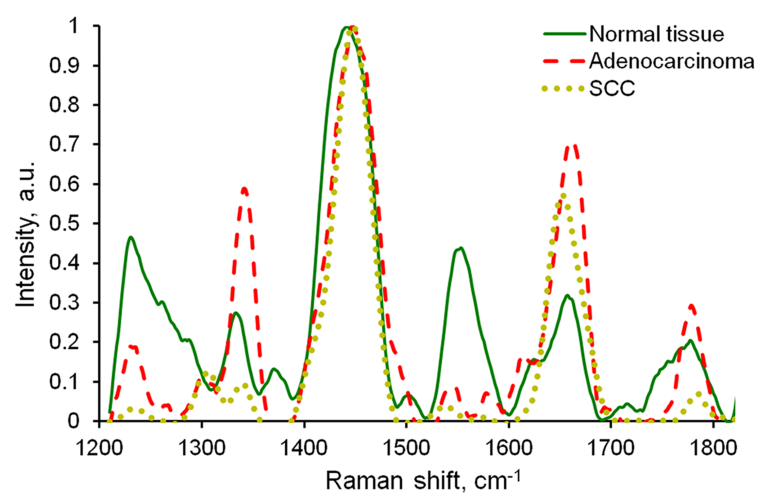

Fig. 2 Normalised ex vivo Raman spectra of healthy lung tissue, adenocarcinoma, and squamous cell carcinoma in the region $1200-1800 \mathrm{~cm}^{-1}$.

For the bands $1200-1240$ and $1540-1580 \mathrm{~cm}^{-1}$ a higher level of intensity is characteristic for healthy tissue in comparison with the adenocarcinoma and squamous cell carcinoma. The intensity value for these bands in healthy tissue can vary from sample to sample, however, its value is always higher than the corresponding spectral intensity of Raman scattering, obtained for a tumor. It is particularly clearly seen for the squamous cell carcinoma, where the signal level only slightly exceeds the level of noise effects. In these bands the vibrations in the following substances are responsible for the appearance of the peak: tryptophan 
(1208, 1552, and $\left.1618 \mathrm{~cm}^{-1}\right)$, phenylalanine (1207 and $\left.1606 \mathrm{~cm}^{-1}\right)$, and porphyrin (1552 and $\left.1618 \mathrm{~cm}^{-1}\right)$ [5, 1114.], which, in turn, is a manifestation of higher content of these substances in healthy tissue as compared to a tumor. This assumption does not agree with the conclusions of Ref. [5] that assume the inverse relative content of the substances in the tumor and in the healthy tissue. The results of recent studies [18] show that the active growth of cancer tumors occurs in the presence of accumulated nerve cells. Particular role in stimulating the growth of cancer neoplasms is played by some receptors of the sympathetic nervous system [18]. Since the functioning of lung is controlled by the vegetative nervous system (the sympathetic nervous system is a part of the vegetative one), the healthy lung tissues adjacent to the tumor region should contain nervous fibres. The nerve cells of the sympathetic system contain such substances as asparagine and glutamine acids that play the role of neuromediators [19]. The Raman spectral bands of asparagine and glutamine acids are located at $1570 \mathrm{~cm}^{-1}$ [20] and can be shifted by $+60 /-40 \mathrm{~cm}^{-1}$. Therefore, the presence of a strong peak at $1550 \mathrm{~cm}^{-1}$ in the normal lung tissue may be caused by accumulation of nerve cells near the pathologic region.

The band $1300-1340 \mathrm{~cm}^{-1}$ is formed by two RS peaks at $1300 \mathrm{~cm}^{-1}$ and $1335 \mathrm{~cm}^{-1}$ that arise due to the vibrational links in collagen, phospholipids, and nucleic acids. The signal level for this band essentially decreases when moving from healthy tissue to the region of squamous cell carcinoma, and for adenocarcinoma the signal remains practically unchanged. In the case of adenocarcinoma the relative fraction of this band in the Raman spectrum is greater due to the "transfer" of some part of the signal to the region of the peak at $1365 \mathrm{~cm}^{-1}$ (deformation vibrations of $\mathrm{COH}$ and $\mathrm{OH}$ in polysaccharides) [21]. Waging vibrations of $\mathrm{CH}_{2}$ and bending vibrations of $\mathrm{CH}$ in tryptophan also contribute to this peak $[17,21]$. The intensity of the peak at $1335 \mathrm{~cm}^{-1}$ for adenocarcinoma increases due to higher concentration of nucleic acids in the tumor [5].

In the lung tissue the vibrations of amid I bonds in proteins having spiral conformation are responsible for the peak at $1650-1670 \mathrm{~cm}^{-1}$. The relative increase of the peak for the tumor tissues can be related to the increase of relative concentrations of protein molecules with $\beta$ folded conformations and conformations of random spirals (statistical balls) [5, 21].

Thus, the specific feature of Raman spectrum and the differences in the intensities of individual bands for the tumor as compared to the healthy lung tissue reflect molecular and cellular changes related to the malignant transformation of the tissue. The essential difference of Raman peak intensity in the bands $1300-1340$ and 1650$1670 \mathrm{~cm}^{-1}$ of the studied samples of tumor and healthy tissue allows the differentiation of the tissue types by means of the Raman spectroscopy. For the determination of the type of the studied tissue the method of phase analysis of intensities in the Raman spectrum of lung tissues is proposed.

\subsection{Double-stage phase method of neoplasm diagnostics}

Most of the known methods of neoplasm Raman spectra analysis [7, 8, 22] are based on the introduction of threshold characteristics of the peak intensities at 1300$1340,1640-1680$ и $1440-1460 \mathrm{~cm}^{-1}$. The sensitivity and specificity of the methods based on the absolute threshold characteristics rarely exceed $80 \%$.

Our studies allow the formulation and proposal of a different method of neoplasm Raman spectra analysis, the method of calculating phase criteria of assessing malignant neoplasms with respect to other types of biological tissues.

The initially recorded Raman spectra are analysed in two stages. At the first stage two phase characteristics are introduced, namely, the ratio of the scattered intensity $I_{1320}$ in the band $1300-1340 \mathrm{~cm}^{-1}$ and $I_{1660}$ in the band $1640-1680 \mathrm{~cm}^{-1}$ with respect to the Raman intensity in the band $1440-1460 \mathrm{~cm}^{-1}$. Then each measurement can be presented as a point in the phase plane $I_{1320}-I_{1660}$ (Fig. 3a). Using the histologic study as an etalon, each experimental point in the phase plane can be attributed to a particular type of tumor or healthy tissue. This makes it possible to introduce a method of successful selection of malignant neoplasms in comparison with the spectra of healthy tissue.

At the second stage of diagnostics the changes of Raman scattering intensities in the neoplasm area were considered with respect to the surrounding healthy tissue in the bands $k=1320,1450$, and $1660 \mathrm{~cm}^{-1}$. For this aim the relative values (indices) of the intensity change were introduced as

$$
\Delta I_{k}=\frac{\left|I_{k}^{(m)}-I_{k}^{(h)}\right|}{I_{k}^{(m)}+I_{k}^{(h)}},
$$

where the superscripts $m$ and $h$ correspond to the intensity values in the malignant area and in the healthy tissue. These three indices compose a phase space, in which every point corresponds to one measurement that can be identified basing on the histological study. The projections on three phase planes (the first with axes $\Delta \mathrm{I}_{1320}$ and $\Delta \mathrm{I}_{1450}$, the second with axes $\Delta \mathrm{I}_{1320}$ and $\Delta \mathrm{I}_{1660}$, and the third with axes $\Delta \mathrm{I}_{1450}$ and $\Delta \mathrm{I}_{1660}$ ) can be directly obtained by the experimental data processing. In ex vivo studies of lung neoplasms the analysis of the phase plane with the coordinates $\Delta \mathrm{I}_{1450}$ and $\Delta \mathrm{I}_{1660}$ was most informative, which allows the separation of the plane into regions, corresponding to different types of neoplasms (Fig. 3b).

The tracing of changes in Raman spectra of neoplasms in comparison with the spectra of healthy tissue in one patient allows precise evaluation of quantitative changes of the concentration of definite substances (markers) in the neoplasm cells. Such assessment, in turn, makes it possible to divide the studied neoplasms into groups with respect to the concentration of the chosen substances. In analogy with 
the first stage, this division is most easily presented in the phase plane.

Figure 3 illustrates the results of the performed studies. To separate the classes in the phase plane, the QDA classifier was used. It is seen that the classes of healthy tissue and those of tumors (adenocarcinoma and squamous cell carcinoma) are well localisable, in spite of some dispersion of the experimental data.

The analysis of Fig. 3a shows that with the use of QDA classifier the sensitivity of lung malignant tumor identification at the first stage amounted to $89.2 \%$, the specificity being equal to $72.9 \%$. However, the first stage of the Raman spectra analysis does not allow the differentiation between the cases of squamous cell carcinoma and adenocarcinoma. In fact, what occurs at the first stage is the separation of malignant neoplasms from benign ones. The second stage (Fig. 3b) allows the separation of the adenocarcinoma cases from those of squamous cell carcinoma and provides the sensitivity value of $90.9 \%$ with the specificity of $71.4 \%$.
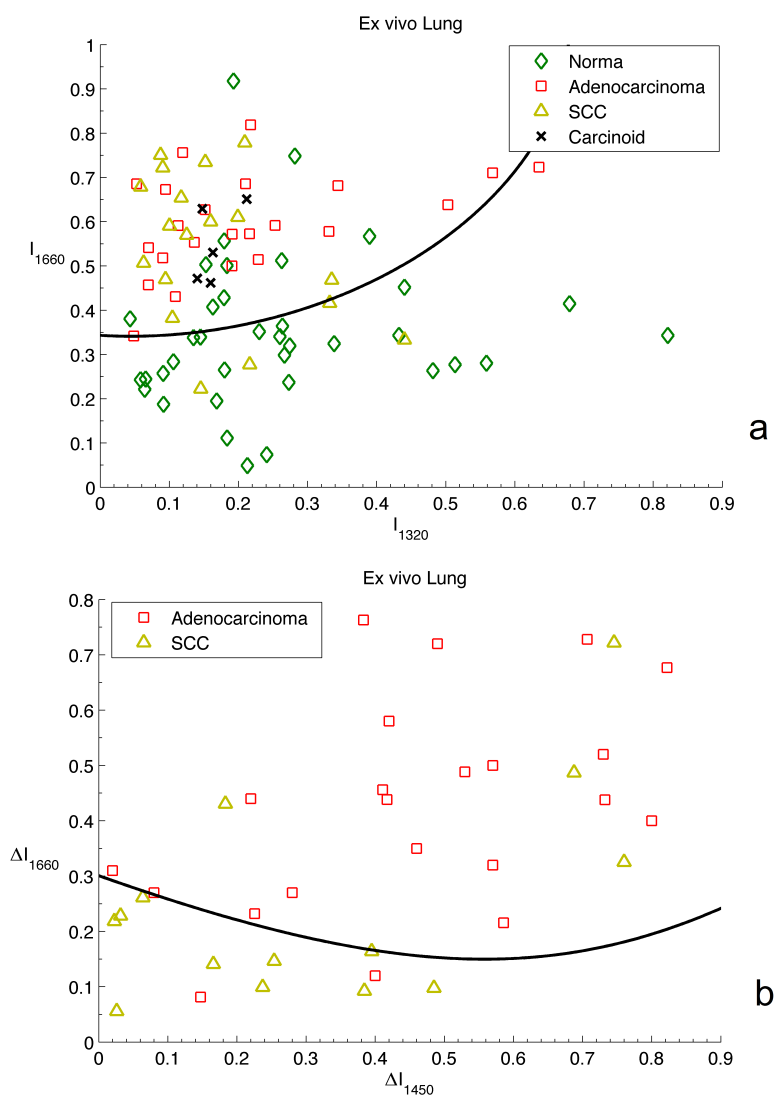

Fig. 3 Classification of lung neoplasm using the doublestage phase method ex vivo: a - differentiation of normal and pathological tissue, $\mathrm{b}$ - differentiation of adenocarcinoma and squamous cell carcinoma.

It is worth noting, that in Fig. 3a the data for carcinoid cancer of lung are additionally shown. The position in the phase plane allows the definite conclusion that the studied type of a tissue is a malignant neoplasm. However, using only the data of Raman spectral analysis it is impossible to assess its type unambiguously even at the second stage. Probably, the latter is due to the small number of studied carcinoid samples.

One of the ways of further improvement of the diagnostics precision is the use of autofluorescence analysis. The registration of the autofluorescence signal is possible with the times much smaller than those required for the recording the Raman spectra. This fact allows the use of autofluorescence analysis for rapid location of the tumor area by the spectral anomaly of the signal.

\subsection{Autofluorescence analysis}

In the chosen range the shape of the autofluorescence spectrum is affected by nucleic acids, fats, haemoglobin, and such proteins as elastin, collagen and keratin. [23]. Characteristic autofluorescence spectra of lung neoplasm and normal tissue are presented in Fig. 4. It is seen that the AF spectrum of the lung tissue demonstrates a nonlinear, exponentially decreasing dependence upon the wavelength.

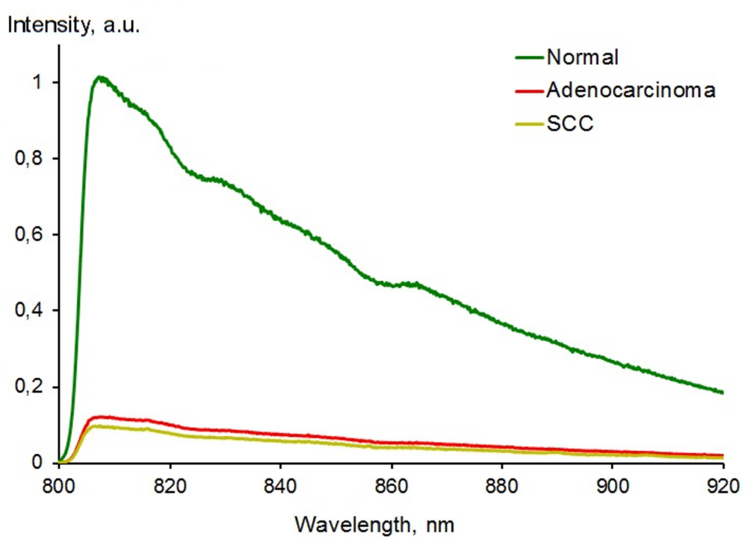

Fig. 4 Typical normalised autofluorescence spectra of lung healthy tissue and neoplasms ex vivo.

By the AF intensity the neoplasms are well differentiated from the healthy lung tissue. The AF level in healthy tissue exceeds the intensity of the signal from adenocarcinoma and squamous cell carcinoma by almost an order of magnitude.

Fig. 5 presents the typical averaged AF spectra of squamous cell carcinoma and normal tissue, plotted with the confidence interval. In this figure one can easily select two non-intersecting regions, located far from each other and corresponding to possible intensity values of the spectra of normal and pathological tissues. The maximal spread of the AF intensity produced by a healthy lung is equal to $20 \%$. The spread increase in the intensity values is due to the steady lowering of the AF signal with time under the continuous exposure of the tissue sample to laser radiation. The longer is the exposure of the sample, the weaker becomes the autofluorescence of the tissue corresponding to tissue photobleaching [12]. 


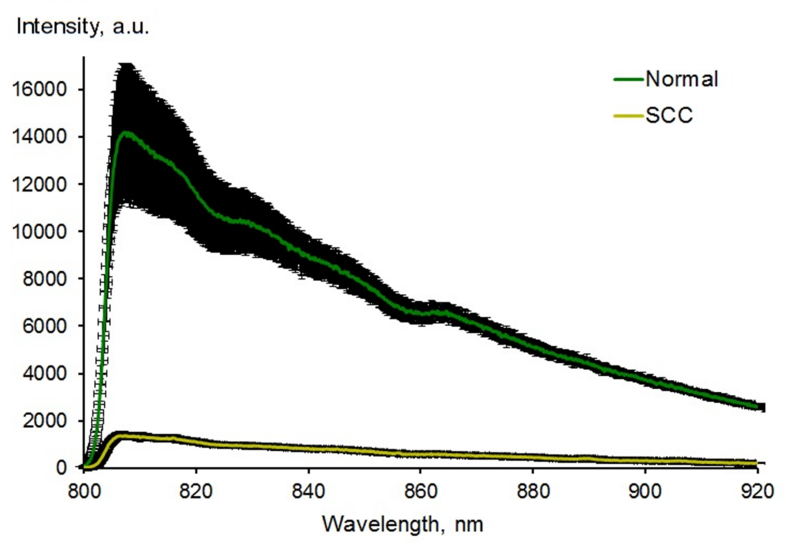

Fig. 5 Typical autofluorescence spectrum of squamous cell carcinoma in comparison with the spectrum of healthy lung tissue.

The AF spectra of four studied carcinoids were by two times smaller in intensity than the mean values of the spectra of adenocarcinoma and squamous cell carcinoma. The AF spectrum intensity of one of the studied samples of carcinoids exceeded the AF value for the normal skin by two times. These data allow the conclusion that using AF analysis one can rapidly reveal the region of spectral anomaly of the tissue, corresponding to the lung oncologic pathology, but without specifying the particular type of the neoplasm.

The characteristic differences in the AF spectra of normal skin and pathologies allow the conclusion that the precision of AF diagnostics of the neoplasm type is smaller than that of Raman spectral analysis. Therefore, using the AF analysis in the IR spectral range solely, it is rather difficult to determine the tumor type reliably. However, the possibility exists to use the AF as an additional method of analysing neoplasms within the complex method of neoplasm type determination together with the analysis of Raman spectrum.

In Ref. [24] it was proved that having $\mathrm{N}$ diagnostic methods (classifiers, weak hypotheses) $w_{i}$, it is possible to construct the method $w_{N+1}=S$ (strong hypothesis), the efficiency of which will exceed those of the methods $w_{i}$. The only necessary condition is that the probability of success for every $w_{i}$ should exceed 0.5 , i.e., should be at least a little better than for random choice from two classes.

One of the autofluorescence analysis application fields can be the fast (the mean acquisition time of $\mathrm{AF}$ signal in our experiments was a few tenths of a second) location of tumor boundaries without determination of the neoplasm type. Fig. 6 presents a map of AF from the sample with squamous cell carcinoma of lung, in which the values of the AF intensity are presented at the points, located along the tumor section at the distance $2.5 \mathrm{~mm}$ from each other. The calculation of the threshold value in the AF map is performed using the Otsu method [25]. The points 1-5 correspond to the tumor and the points 6-8 to the healthy lung tissue.

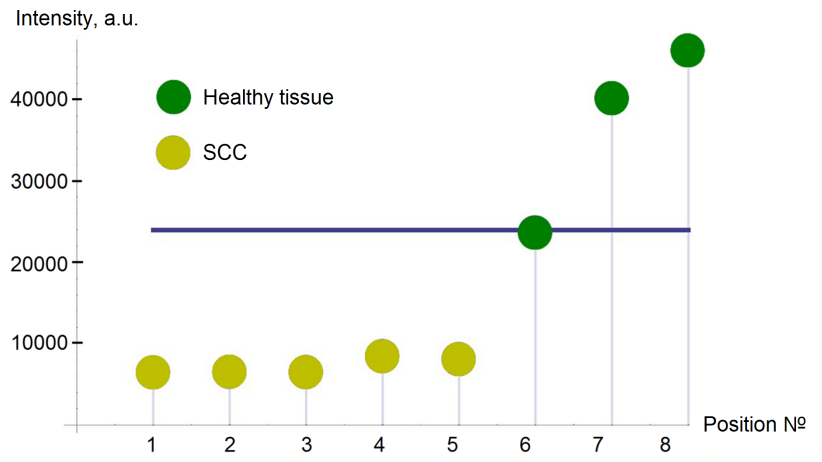

Fig. 6 The AF map of squamous cell carcinoma (SCC) in the healthy lung tissue.

\section{Conclusion}

The first results of the combined use of Raman spectroscopy and autofluorescence analysis demonstrate high efficiency of the proposed method for the differential diagnostics of lung malignant neoplasms The high sensitivity and speed of performing the study indicate the high potential capabilities of the method.

The efficiency of the proposed double-stage method of Raman diagnostics of lung neoplasms is, upon the average, by $5-10 \%$ higher in comparison with other methods of diagnostics $[5,8]$, based on the analysis of Raman scattering. The method allows not only the detection of the pathology, but also the determination of the particular neoplasm type. It is important that the individual characteristics of the Raman spectrum obtained for the neoplasm and the healthy tissue, are compared in each patient (the second stage of the study). This approach allows individualisation of the neoplasm analysis method. High sensitivity indicators are particularly important in the organisation of mass screening of neoplasms during the preventive examinations, because in this case the method yields no false negative results, and the probability of missing the disease is essentially reduced.

The method of Raman spectroscopy has an advantage in sensitivity and specificity of determination of the particular type of neoplasm, but yields to the AF method in the speed of the signal procession (in our experiments the acquisition of signal during 2-3 minutes at each measurement point was required to reduce the noise effect). The combined use of AF and RS spectroscopy allows fast detection of the pathology presence using the AF determination of its boundary, and the analysis of the neoplasm type can be performed already inside the selected area, using the RS method.

\section{Acknowledgments}

This research was supported by the Ministry of Education and Science of the Russian Federation. 